\title{
Countermeasures to Asymptomatic and Presymptomatic Transmission of COVID-19 are Matter
}

\author{
Yoojin Park ${ }^{1 *}$, In Sil Huh ${ }^{1 *}$, Cho Ryok Kang ${ }^{2}$, Jaekyung Lee ${ }^{3}$, Sung-il Cho ${ }^{4}$, Hyon Jin, Ham ${ }^{2}$, \\ Hyo Yeon $\mathrm{Yu}^{5}$, Jung il Kim², Yong-Tae Kwon ${ }^{6}$, Sun Hee Yang ${ }^{7}$, Baeg Ju Na ${ }^{* * *}$, Jin Yong Lee ${ }^{9,10,11^{* *}}$ \\ and Seoul Metropolitan Government COVID-19 Rapid Response (SCoRR) Team \\ ${ }^{1}$ Seoul Center for Infectious Disease Control and Prevention, \\ 2 Disease Control and Prevention Division, Seoul Metropolitan Government, \\ ${ }^{3}$ Department of Internal Medicine, Seoul Metropolitan Government-Seoul National University Boramae Medical Center, \\ ${ }^{4}$ Graduate School of Public Health, Seoul National University, \\ ${ }^{5}$ Special Judicial Police Bureau for public Safety, Seoul Metropolitan Government, \\ ${ }^{6}$ Director of Public Health Division, Gangnam Public Health Center, \\ ${ }^{7}$ Director of Health Management Division, Seocho Public Health Center, \\ ${ }^{8}$ Graduate School of Urban Public Health, University of Seoul, \\ 9 HIRA Research Institute, Health Insurance Review \& Assessment Service, \\ ${ }^{10}$ Public Healthcare Center, Seoul National University Hospital, \\ ${ }^{11}$ Department of Health Policy and Management, Seoul National University College of Medicine
}

*Ms. Yoojin Park, In Sil Huh contributed equally to this article

\section{Correspondence to Baeg Ju Na}

Graduate School of Urban Public Health, University of Seoul, 110, 163 Seoulsiripdaero, Dongdaemun-gu, Seoul 02504, Republic of Korea.

E-mail: baegju.na@seoul.go.kr

Jin Yong Lee, MD, PhD, MHA.

Department of Public Health and Community Medicine, Seoul Metropolitan Government-Seoul National University Boramae Medical Center, 20 Boramae-ro 5-gil, Dongjak-gu, Seoul 07061, South Korea.

E-mail: jylee2000@gmail.com 
Several studies have been reported the possibility of severe acute respiratory syndrome coronavirus 2 (SARS-CoV-2) transmission from persons who test positive, but symptoms never develop (asymptomatic) or who are test positive before symptom onset (presymptomatic) [1-3]. In particular, asymptomatic transmission has been identified in healthcare workers, and in several countries, its prevalence in this population is higher than that in the general population [4-5]. The effective reproductive number (RO) of asymptomatic carriers has been estimated to be between 5.5 and 25.4 with a 15.4 point estimate [6]. This raises concerns about asymptomatic carriers prolonging the spread of COVID-19, as symptom-based screening could miss asymptomatic infections. Therefore, new strategies need to be considered to effectively monitor and help control the outbreak. This case study presents an outbreak of COVID-19 in healthcare professionals that involved asymptomatic and presymptomatic carriers.

Nurse A1, who worked in the operating room of Samsung Medical Center, had symptoms of mild fever, sore throat, sputum, and cough starting on May 16, 2020. She was confirmed to be infected with severe acute respiratory syndrome coronavirus 2 (SARS-CoV-2) on May 18, 2020 (Ct value: E/RdRp 14.78/15.37). Meetings with hospital staff, the Seoul Metropolitan Government COVID-19 Rapid Response (SCORR) Team, and the public health center were carried out in an external building situation room, maintaining a clean zone environment. Unlike the locking down of a high-density working environment where the route of infection is unknown, epidemiological and contact tracing were conducted in accordance with the stepwise hospital infection response protocol to prevent medical vacancies $[7,8]$. Three hundred and sixteen individuals including medical staff, discharged patients, and hospitalized patients who were exposed to the nurse were instructed to self-isolate for 14 days (including those who had negative diagnostic tests). Six hundred and sixty-three hospital staff members were subject to active monitoring twice a day for fever within the hospital. Four hundred and ninety-one employees and dormitory residents underwent SARS-CoV-2 tests as a precautionary measure; these individuals also received health education. COVID-19 was confirmed in three nurses (A2 with symptoms, E/RdRp 15.82/17.25, as well as A3 E/RdRp 15.75/16.91 and AB1 E/RdRp 25.42/26.22, asymptomatic at diagnosis), who had had contact with a symptomatic case, translating to a secondary attack rate of $0.83 \%$ (3/363) (Figure 1 ).

For the four confirmed cases, the source of infection was unknown. To interrupt asymptomatic transmission, the hospital and SCoRR team took a more active approach, and a text message was sent to about 60,000 discharged patients informing them to get tested for COVID-19. Moreover, a survey of individuals' whereabouts during the 14-day incubation was conducted to confirm infection route. It was confirmed that AB1 was in contact with a group of five friends at A Pub near Gangnam station on May 10, 2020; two of the six attendees at this gathering were further confirmed to have COVID-19 (B1: Symptoms with mild fever, E/RdRp 20.29/21.86; B2: Asymptomatic, E/RdRp 24.66/26.15). In consideration of Ct value and exposure history at the multiuse facility, it was determined that there was asymptomatic spread in the Samsung Medical Center. 


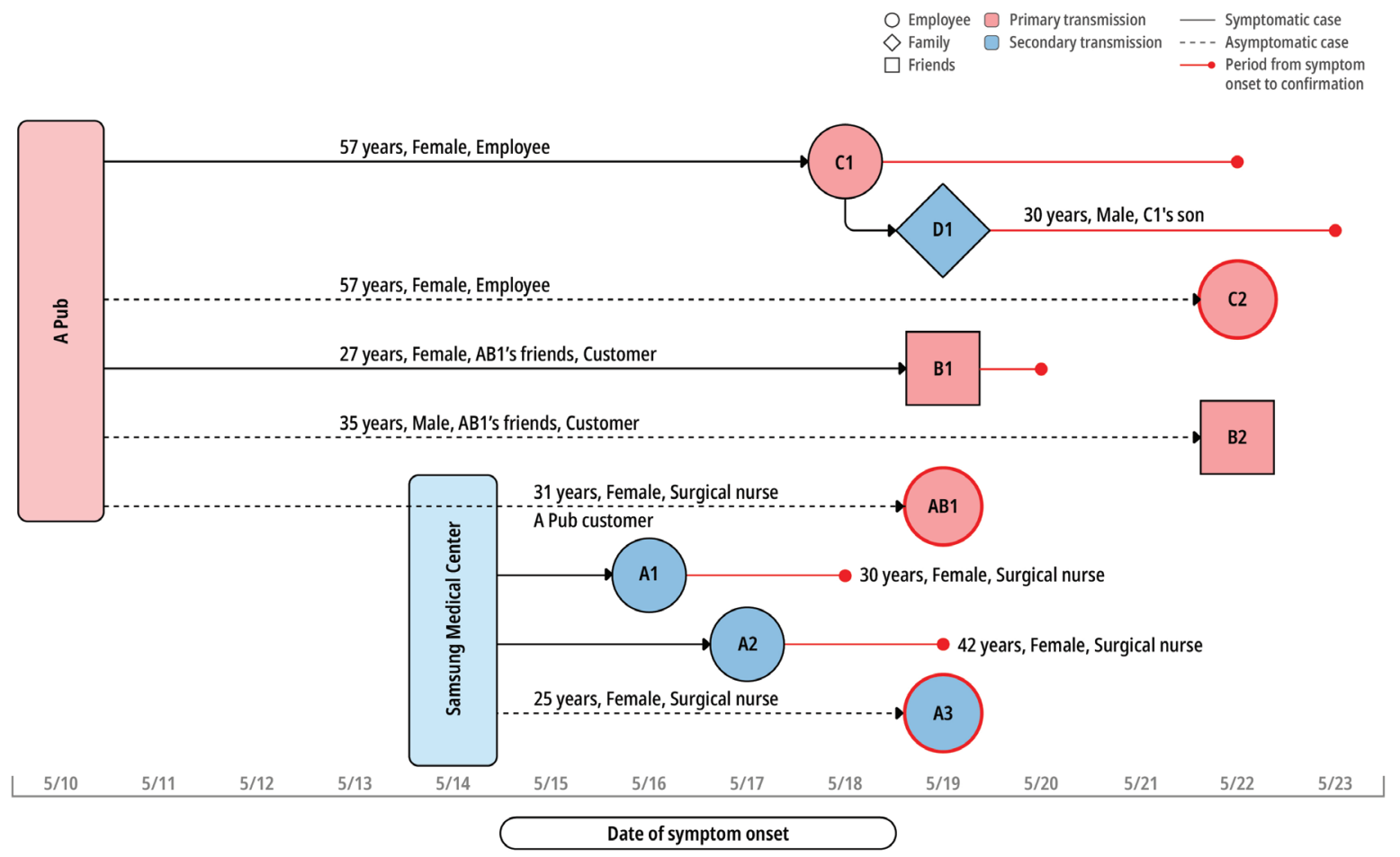

<Figure 1> Case map of confirmed COVID-19 cases associated cases associated with c lusters occurred in Samsung Medical Center and A Pub.

Immediately upon discovering asymptomatic transmission, an environmental evaluation of pubs near Gangnam Station - those without ventilation and active customer movements owing to "all-you-can-drink" schemes - was conducted. Of the employees, two servers tested positive (C1: Symptoms of dry sweat and shivering, E/RdRp 32.14/32.97; C2: Asymptomatic, E/RdRp 31.74/31.95). The city of Seoul and Gangnam-gu then sent out a disaster alert text to all people who visited the pub on May 10, 2020, regardless of the presence of symptoms. Through this, it was confirmed that D1 (Symptoms of sputum, stuffy nose, E/RdRp 18.12/20.30), a family member of employee C1, was confirmed to have COVID-19. The serial interval from C1 to D1 was a 1 day incubation period (4 days, SD 2-14), suggesting viral shedding before symptoms occur, leading to substantial presymptomatic transmission (Table 1) [9]. 
$<$ Table 1> Characteristic of COVID-19 cases associated with clusters occurred in Samsung Medical Center and A Pub.

\begin{tabular}{|c|c|c|c|c|c|c|c|c|c|}
\hline \multirow{2}{*}{ Cluster } & \multirow{2}{*}{$\begin{array}{l}\text { Patient } \\
\text { no. }\end{array}$} & \multirow{2}{*}{ Sex } & \multirow{2}{*}{ Age } & \multirow{2}{*}{$\begin{array}{l}\text { Symptom } \\
\text { onset }\end{array}$} & \multirow{2}{*}{ Symptom } & \multirow{2}{*}{$\begin{array}{c}\text { Confirmed } \\
\text { date }\end{array}$} & \multicolumn{2}{|c|}{ Ct value } & \multirow{2}{*}{ Exposure history } \\
\hline & & & & & & & $E$ & $R d R p$ & \\
\hline \multirow{4}{*}{$\begin{array}{c}\text { Samsung } \\
\text { Medical } \\
\text { Hospital }\end{array}$} & A1 & & & $5 / 16$ & Headache & $5 / 18$ & 14.78 & 15.37 & $\begin{array}{l}\text { Surgical ward C with AB1; } \\
\text { Hospital S }\end{array}$ \\
\hline & $\mathrm{A} 2$ & $\mathrm{~F}$ & 42 & $5 / 17$ & $\begin{array}{l}\text { Headache, low } \\
\text { grade fever }\end{array}$ & $5 / 19$ & 15.82 & 17.25 & $\begin{array}{l}\text { Surgical ward C with AB1; } \\
\text { Hospital S }\end{array}$ \\
\hline & A3 & $\mathrm{F}$ & 25 & - & Asymptomatic & $5 / 19$ & 15.75 & 16.91 & $\begin{array}{l}\text { Surgical ward C with AB1; } \\
\text { Hospital S }\end{array}$ \\
\hline & AB1 & $\mathrm{F}$ & 31 & - & Asymptomatic & $5 / 19$ & 25.42 & 26.22 & $\begin{array}{l}\text { Gathering with B1 \& B2; } \\
\text { Pub A, May } 10\end{array}$ \\
\hline \multirow{5}{*}{ A Pub } & B1 & $\mathrm{F}$ & 27 & $5 / 19$ & Low grade fever & $5 / 20$ & 22.29 & 21.86 & $\begin{array}{l}\text { Gathering with AB1 \& B2; } \\
\text { Pub A, May } 10\end{array}$ \\
\hline & B2 & $\mathrm{M}$ & 35 & - & Asymptomatic & $5 / 22$ & 24.66 & 26.15 & $\begin{array}{l}\text { Gathering with AB1 \& B2; } \\
\text { Pub A, May } 10\end{array}$ \\
\hline & $\mathrm{C} 1$ & $\mathrm{~F}$ & 57 & $5 / 18$ & Cold sweat & $5 / 22$ & 32.14 & 32.97 & Employee working in Pub A \\
\hline & $\mathrm{C} 2$ & $\mathrm{~F}$ & 57 & - & Asymptomatic & $5 / 22$ & 31.74 & 31.95 & Employee working in Pub A \\
\hline & D1 & $\mathrm{M}$ & 30 & $5 / 19$ & $\begin{array}{l}\text { Sputum, } \\
\text { stuffy nose }\end{array}$ & $5 / 23$ & 18.12 & 20.30 & Son of C1 \\
\hline
\end{tabular}

In order to flatten the curve of COVID-19, SMG have implemented extensive diagnostic tests through epidemiological investigations of confirmed patients $[7,8]$. However, because of the spread of asymptomatic or presymptomatic COVID-19 across Seoul, SMG developed unprecedented countermeasures of preemptive testing for volunteers or vulnerable people without symptoms, regardless of exposure history. The preemptive committee also evaluated risk rates for selected targeted groups such as health care workers and high school students residing in dormitories. Pooled test was conducted for preemptive testing, where grouped 3-4 individual samples are tested in a single tube by molecular biological detection methods. Individual samples are tested again only if the result of pooled test is positive.

In conclusion, this case study demonstrates that asymptomatic and pre-symptomatic transmission occurred in Seoul, and that wide-range testing and preemptive investigation strategies were effective in preventing the spread of SARSCoV-2 in the community by identifying asymptomatic and pre-symptomatic carriers. 


\section{References}

1. Melissa M Arons, Kelly M Hatfield, Sujan C Reddy, Anne Kimball, Allison James, et al., Presymptomatic SARS-CoV-2 infections and transmission in a skilled nursing facility. N Engl J Med. 2020:28;382(22):2081-2090

2. Rachael Pung, Calvin J Chiew, Barnaby E Young, Sarah Chin, Mark I-C Chen, et al., Investigation of three clusters of COVID-19 in Singapore: implications for surveillance and response measures. Lancet. 2020:28;395(10229):1039-1046

3. Furukawa N, J Brooks, J Sobel. Evidence Supporting Transmission of Severe Acute Respiratory Syndrome Coronavirus 2 While Presymptomatic or Asymptomatic. Emerging infectious diseases. 2020: 26(7).

4. Monica Gandhi, Deborah S Yokoe, Diane V Havlir. Asymptomatic transmission, the Achilles' heel of current strategies to control COVID-19. N Engl J Med. 2020:28;382(22):2158-2160.

5. Li Q, Guan X, Wu P, Wang X, Zhou L, Tong Y et al. Early transmission dynamics in Wuhan, China, of novel coronavirus-infected pneumonia. New England Journal of Medicine.2020

6. Aguilar JB, Faust JS, Westafer LM., Gutierrez JB. Investigating the impact of asymptomatic carriers on COVID-19 transmission. medRxiv, 2020

7. Na BJ, Yoojin Park, In Sil Huh, Cho Ryok Kang, Jaekyung Lee, Jin Yong Lee. Seventy-two Hours, Targeting Time from First COVID-19 Symptom Onset to Hospitalization. J Korean Med Sci. 2020:25; 35(20): e192.

8. Park SY, Kim YM, Yi S, Lee S, Na BJ, Kim CB, Kim JI, et al., Coronavirus Disease Outbreak in Call Center, South Korea. Emerg Infect Dis. 2020;26(8):1666-1670

9. Nishiura H, Linton NM, Akhmetzhanov AR. Serial interval of novel coronavirus (COVID-19) infections. International journal of infectious diseases, 2020:93; 284-286. 
